\title{
UNA APROXIMACIÓN AL NARRATIVISMO DE ALASDAIR MACINTYRE EN "ETHICS IN THE CONFLICTS OF MODERNITY. AN ESSAY ON DESIRE, PRACTICAL REASONING AND NARRATIVE"
}

\author{
A CRITICAL APPROACH TO ALASDAIR MACINTYRE'S NARRATIVISM IN “ETHICS IN \\ THE CONFLICTS OF MODERNITY. NA ESSAY ON DESIRE, PRACTICAL REASONING \\ AND NARRATIVE”
}

UMA APROXIMAÇÃO AO NARRATIVISMO DE ALASDAIR MACINTYRE EM "ETHICS IN THE CONFLICTS OF MODERNITY. NA ESSAY ON DESIRE, PRACTICAL REASONING AND NARRATIVE”

\author{
María Agustina Juri \\ (Universidad Nacional de Cuyo) \\ agustina.juri@gmail.com
}

Recibido: 02/12/2019

Aprobado: 09/01/2020

\begin{abstract}
RESUMEN
En el presente trabajo nos proponemos analizar la nueva aproximación al narrativismo que realiza Alasdair MacIntyre en su último libro: Ethics in the Conflicts of Modernity. An Essay on Desire, Practical Reasoning and Narrative (2016). En primer lugar, realizamos un recorrido histórico por las obras más significativas en las que MacIntyre desarrolla su visión del narrativismo en primera instancia: After Virtue. A Study in Moral Theory (1981), Whose Justice, Which Rationality? (1988) y Three Rival Versions of Moral Enquiry. Enciclopaedia, Genealogy and Tradition (1990). En segundo lugar, intentamos explorar la reincidente problemática de la narratividad en la obra del 2016 para explicar cómo exhibe un agente neoaristotélico la racionalidad y cómo justifica sus juicios a través de una narrativa. Además, exploramos por qué la narrativa cobra más protagonismo en esta obra que la noción de tradición y evidencia una mayor relación con el telos de la vida humana. También, señalamos una reafirmación de la posición macinteryana desde el aristotelismo frente a los ahora denominados expresivistas.
\end{abstract}

Palabras clave: Narrativismo. Tradición. Telos. Acciones humanas.

\begin{abstract}
The aim of this article consists in analyzing Alasdair MacIntyre's new approach to narrativism in his most recent work: Ethics in the Conflicts of Modernity An Essay on Desire, Practical Reasoning and Narrative (2016). In the first place, we make a historical review through the most relevant MacIntyre's Works on narrativism: After Virtue. A Study in Moral Theory (1981), Whose Justice, Which Rationality? (1988) y Three Rival Versions of Moral Enquiry. Enciclopaedia, Genealogy and Tradition (1990). Secondly, we explore the problem of narrativity that appears again in the work of 2016 to explain how does a Neoaristotelian agent exhibit his own rationality and how does he justify his judgements through a narrative. Also, we explore why narrative is more important than the concept of
\end{abstract}


Tradition in this work and is more related to the telos of human life. Finally, we emphasize the the macintyrean position against the now called expressivists.

Keywords: Narrativism. Tradition. Telos. Human Actions.

\section{RESUMO}

Neste artigo, propomos analisar a nova abordagem ao narrativismo que Alasdair MacIntyre faz em seu último livro: Ética nos conflitos da modernidade. Ensaio sobre Desejo, Raciocínio Prático e Narrativa (2016). Em primeiro lugar, fizemos um tour histórico pelas obras mais significativas nas quais MacIntyre desenvolve sua visão do narrativismo em primeiro lugar: After Virtue. Um estudo em teoria moral (1981), cuja justiça, que racionalidade? (1988) e três versões rivais do inquérito moral. Encyclopaedia, Genealogy and Tradition (1990). Em segundo lugar, tentamos explorar a reincidência problemática da narratividade no trabalho de 2016 para explicar como um agente neo-aristotélico exibe racionalidade e como ele justifica seus julgamentos por meio de uma narrativa. Também exploramos porque a narrativa assume mais destaque neste trabalho do que a noção de tradição e mostra uma relação maior com o télos da vida humana. Também apontamos uma reafirmação da posição de macintyreana do aristotelianismo contra os agora chamados expressivistas.

Palavras chaves: Narrativismo. Tradição. Télos. Ações humanas.

\section{Case History}

Imprisoned in a portrait of herself

Painted by someone else, she slashed the canvas

And then ran crying to her looking-glass

To find a true reflection of herself,

And -finding none- could not tell who she was.

Not glass, not canvas could restore her knowledge,

Only another's face in which to see

That emptiness itself can be an image

Of victories over false identities

Without that face he waits in lonely rage.

Alasdair MacIntyre ${ }^{1}$

Que la narratividad ha sido un tema constante en la obra de Alasdair MacIntyre desde, al menos 1977, hasta la actualidad, es indudable. Numerosos artículos, libros y conferencias del escocés así lo demuestran. Esta característica tan particular que, junto con otras propiedades, hacen del pensamiento de nuestro autor un fenómeno verdaderamente original que ha ido tomando diversas formas a lo largo del tiempo.

En conversaciones mantenidas con el profesor MacIntyre durante el otoño del año 2018, en el $D e$ Nicola Center for Ethics and Culture de la University of Notre Dame, Indiana, Estados Unidos, se nos transmitió dos preguntas iluminadoras que resumen algunas de sus inquietudes relacionadas con la narratividad. La primera pregunta es ¿por qué MacIntyre toma las acciones humanas y las investigaciones como inteligibles sólo en términos de (a) narrativas y (b) tradiciones? La segunda pregunta es ¿cómo exhibe un agente la racionalidad y justifica sus juicios a través de una narrativa?

${ }^{1}$ Tuvimos acceso a este poema de los años ‘60 que funciona como epígrafe del presente trabajo consultando el archivo MacIntyre del De Nicola Center for Ethics and Culture de la University of Notre Dame, Indiana, Estados Unidos. 
La respuesta a la primera inquietud podríamos encontrarla en un corpus que nosotros, junto con el mismo MacIntyre (Borradori, 1991), David Lorenzo Izquierdo (2007) y otros analistas, reconocemos como parte un proyecto unitario de raíz aristotélica: After Virtue. A Study in Moral Theory (1981), Whose Justice, Which Rationality? (1988) y Three Rival Versions of Moral Enquiry. Enciclopaedia, Genealogy and Tradition (1990). Se podría afirmar, a partir de la lectura de este corpus, que MacIntyre lleva a cabo en él un proyecto de renovación epistemológica de la ética al mismo tiempo que una actualización de la tradición tomista aristotélica $^{2}$, tema que nos ocupa actualmente, y que, por cuestiones de extensión, no podremos desarrollar con profundidad en este trabajo, por lo que queda pendiente para otras publicaciones.

Por otro lado, nos atreveríamos a sostener que el segundo interrogante ha dado lugar a una profundización en el concepto de narratividad en Ethics in the Conflicts of Modernity. An Essay on Desire, Practical Reasoning and Narrative (2016), el libro más reciente de MacIntyre. Allí nuestro autor, a diferencia del corpus antes mencionado en el que transita la narratividad principalmente como método, recurre a la narratividad como núcleo temático, la liga estrechamente a la formulación del juicio práctico e incluso responde a las objeciones a la narratividad en general de parte de Galen Strawson, también a las críticas a su propio uso de la narrativa de parte de László Tengelyi, y concluye su ensayo con cuatro narraciones de vidas de razonadores prácticos -para él-, ejemplares.

En este trabajo nos proponemos analizar a fondo el concepto de narratividad en Ethics in the Conflicts of Modernity y comenzaremos con la comparación de los diversos usos y significados que MacIntyre le otorga a la narratividad a lo largo del tiempo, para luego tratar de dilucidar algunas cuestiones, a nuestro juicio, relevantes: a) por qué en esta obra se centra más en el concepto de narrativa y no tanto en el de Tradición; b) por qué considera que la narratividad es inherente a la justificación racional de los agentes tomistas aristotélicos; c) cuál es el argumento último que legitima la narratividad como el principio de inteligibilidad de las acciones humanas.

\section{Primera cuestión:}

\section{¿Por qué MacIntyre toma las acciones humanas y las investigaciones como inteligibles sólo en términos de (a) narrativas y (b) tradiciones?}

Cabe señalar que MacIntyre divide su propio pensamiento en tres etapas. En primer lugar, el periodo de la metaética (1949-1971) impregnado de marxismo, freudismo y filosofía analítica. Luego, al descubrir que las bases epistemológicas del materialismo dialéctico no eran suficientes para combatir el liberalismo y de decepcionarse del marxismo político, comenzó la etapa de autocrítica y revisión (1971-1977). Más adelante, MacIntyre comienza lo que él reconoce como la tercera etapa que se extiende desde 1977 hasta la actualidad, en la que hace un giro radical hacia el aristotelismo y afirma, a partir de allí, "he estado comprometido en un único proyecto en el que After Virtue, Whose Justice? Which Rationality? y Three Rival Versions of Moral Enquiry son centrales, un proyecto descrito por uno de mis colegas como el de escribir una historia de la ética interminablemente larga" (Knight, 1998: 267)

Fue en ese mismo año que inauguró la tercera etapa con un novedoso artículo titulado Epistemological Crises, Dramatic Narrative and the Philosophy of Science en donde intenta rescatar la dimensión histórica de la tarea investigativa de los filósofos, hecho que ellos mismos suelen obviar, sin contar con la dimensión moral, que implica esta actividad que debe tender a la búsqueda de la verdad ${ }^{3}$. Además, señala que tanto la historia de la epistemología cuanto la historia de la ética no son tenidas en cuenta como narrativas, en afán de eludir su carácter evaluativo, en el que hay buenos o malos personajes que

\footnotetext{
${ }^{2}$ Expresamos el término 'tomista aristotélico' y no 'aristotélico tomista', ya que MacIntyre en diversas obras y personalmente en una reunión en octubre de 2018, nos ha aclarado que se considera a sí mismo aristotélico en primer lugar y, en segundo lugar, seguidor de Santo Tomás de Aquino por ser quien mejor ha desarrollado las tesis aristotélicas, y no al revés.

${ }^{3}$ En relación con el tema de la verdad, se puede consultar también MacIntyre, A. (1978) Objectivity in Morality and Objectivity in Science. En H. Tristram Englehardt, Jr \& Daniel Callahan (eds.), Morals, Science and Sociality. Hastings Center. pp. 21-39
} 
producen resultados felices o infelices. Tanto es así, que MacIntyre considera que el progreso epistemológico consiste en la construcción y reconstrucción de narrativas, y que las crisis epistemológicas constituyen momentos oportunos para estas reconstrucciones.

Otro concepto fundamental que va a desarrollar extensamente en el corpus antes mencionado es el de tradición de investigación racional, ya que ésta aporta el contexto en el que se desarrolla determinada filosofía. Quien más resistencia ha expresado a esta noción en la historia de la filosofía ha sido René Descartes, al reducir toda certeza al cogito. Por eso, es a él a quien MacIntyre refuta con firmeza en el artículo de 1977. El escocés critica las oposiciones modernas de tradición-razón y de tradiciónrevolución aduciendo que son las tradiciones las que conllevan la razón, y que son las tradiciones las que necesitan, en algunos momentos -siguiendo a Kuhn-, de las revoluciones científicas para continuar. Sostiene, además, que la narrativa dramática es la forma apropiada para la comprensión de la acción humana y que la racionalidad de la investigación científica depende de la razón histórica, esto es, de la narrativa. En suma, en este primer esbozo de la narratividad, MacIntyre sostiene que las narrativas dramáticas, con las teorías como protagonistas y con pretensión de verdad histórica, son capaces de hacer inteligibles las diversas tradiciones científicas para, de esa forma, poder evaluarlas. Otra idea central que se va a mantener hasta la actualidad es el falsacionismo popperiano.

Augusto Hortal Alonso (1998) nos recuerda que en la primera mitad del siglo XX la ética había sido rechazada por parte del positivismo lógico. Wittgenstein, por ejemplo, la situaba en la esfera de lo inefable. Por lo tanto, la teoría moral quedó en manos del existencialismo, por un lado, que reducía la ética a la autenticidad, y el neomarxismo, por otro, que ponderaba como ideológica toda ética que no contribuyera a reproducir las prácticas del cambio revolucionario de las estructuras opresoras en pos de la liberación. Hasta finales de los años sesenta, la ética, junto a otras disciplinas, estuvo marginada, a excepción de los desarrollos emotivistas de Charles Leslie Stevenson, hasta la aparición de A Theory of Justice de John Rawls en 1971 y la Teoría de la acción comunicativa de Habermas en 1976. El libro de Rawls promovía el universalismo deontológico, siguiendo a Kant, filosofía predominante hasta los años 90.

Hacia 1981 con la publicación de After Virtue, MacIntyre diagnostica una crisis epistemológica en el campo de la ética y la consiguiente decadencia de la cultura contemporánea. Reconoce la existencia de versiones de teorías morales que no dialogan entre sí que han dado como resultado una reducción de la ética a preferencias subjetivas y arbitrarias, es decir, el emotivismo.

Para superar esta crisis, MacIntyre desarrolla un argumento, entendiendo este término como una mezcla de argumentación filosófica y narración dramática.

\footnotetext{
"Se nos narra una historia intelectual y moral: la decadencia de la moralidad en la edad moderna y las posibilidades que quedan para un posible resurgir de ella. Pero, por otra parte, 'argumento' también es 'alegato racional', en continuidad con las típicas discusiones de la filosofía analítica. El afán de argumentar, no siempre de manera exhaustiva, está detrás de cada una de las principales, arriesgadas y provocativas afirmaciones de MacIntyre en Tras la virtud" (Hortal, 1998: 2-3).
}

De esta manera, MacIntyre traza un proyecto de renovación epistemológica de la ética sobre la base de dos ideas fundamentales acerca de la narrativa que expondremos a continuación.

En primer lugar, podría afirmarse que el narrativismo de MacIntyre se funda en una particular concepción antropológica. El escocés define al hombre tanto en sus acciones como en sus prácticas, y también en sus ficciones, como un animal que cuenta historias (MacIntyre, 1981: 216). Gracias a las historias de su familia, de su ciudad, de su país puede forjar su identidad, ya que en su vida cobran unidad. La definición aristotélica de la trama como inicio, desarrollo y fin es lo que le permite al hombre organizar su propia experiencia, lo que le permite hacer inteligibles sus acciones y su propia vida. Sin embargo, las acciones han sido descontextualizadas sistemáticamente por la filosofía moral académica en un proceso que comienza en la Modernidad y que desemboca en la relativización de los valores morales en la Posmodernidad. En suma, la base antropológica macinteryana se basa en la relación de inteligibilidad entre acción y narrativa. 
La narrativa sirve para hacer inteligible lo que la gente hace o dice, pero no sólo se reduce a eso. En el epílogo a la 2 da edición de After Virtue, MacIntyre aclara que la relación próxima entre filosofía e historia que posibilita la narratividad, puede hacer inteligible cómo llega a plantearse una teoría y en qué tipo de situaciones ello es relevante, tanto para comprenderla como para valorarla. Si nos preguntamos por qué la gente actúa de determinada manera, la única forma de explicarlo es mediante una narración. Y esta narración tiene, por supuesto, una dimensión histórico-social en la que hay narrativas históricas mayores que otras. Además, la narrativa implica recuperar un enclave espaciotemporal perdido por el universalismo abstracto moderno que impedía un acercamiento a la realidad de la acción.

En segundo lugar, en un plano epistemológico, MacIntyre pasa a considerar que además de representar las acciones humanas, la narrativa da forma a la representación de las tradiciones de investigación racional. En un entrevista para la revista Cogito (Knight, 1998: 270), el escocés afirma "rational enquiry is itself always tradition dependent". En Whose Justice? Which Rationality? MacIntyre continúa la narración de la historia de las tres tradiciones de investigación racional de la ética, a saber, la correspondiente a la Modernidad o Enciclopédica, la tradición de la Genealogía nietzscheana y la Tradición de las virtudes, a las que añade, en este texto, una cuarta: la tradición del liberalismo ${ }^{4}$.

En el capítulo XVIII de dicha obra, MacIntyre enumera las características propias de la tradición de investigación racional. Indica allí que siempre se da en un contexto social y moral, siempre se desarrolla en el seno de una comunidad, y que la historia narrativa de las tradiciones incluye el relato de las investigaciones y del debate que se da dentro de ella y las discusiones con sus rivales. Además, ellas son una narración siempre inconclusa, susceptible de perfeccionarse con el paso del tiempo.

Las tradiciones se configuran como continuidades en conflicto, que sobreviven en el tiempo de forma dialéctica ad intra y ad extra, en la medida en que pueden dar respuestas válidas a las objeciones que se les presentan. Es por esto que MacIntyre afirma que el tipo de justificación racional que alcanzan es dialéctica e histórica, y una vez que han superado una crisis epistemológica, sus protagonistas pueden reescribir la historia de dicha tradición de una forma más profunda y acabada. De este modo, la comprensión del pasado posibilita la comprensión de las posibilidades futuras.

A diferencia de la inalcanzable pretensión moderna de un punto de vista neutro e independiente de todas las tradiciones, MacIntyre sostiene que todo pensamiento surge en el seno de una tradición, y que es allí desde donde se debe buscar la verdad que es intemporal, entendida como la adecuación del intelecto con su objeto. Podemos observar, entonces, que la única forma de hacer inteligible una tradición de investigación racional es a partir de la narrativa histórica que cuente sus victorias y fracasos en el diálogo con sus rivales.

Ya en Three Rival Versions of Moral Enquiry MacIntyre se propone concretamente hacer productivos los desacuerdos morales entre las tres versiones. Mientras la posición genealogista sostiene que la narrativa histórica tiene una función distorsionante y funciona como un instrumento de represión, la tradición de la Enciclopedia funda su narrativa en una historia unificada del progreso. (MacIntyre, 1990: 88) Resulta preciso señalar que un año después de la publicación de After Virtue MacIntyre se convierte al catolicismo y comienza a adentrarse en el tomismo. Tanto es así que ya en 1990 afirmará que, a diferencia de las dos tradiciones ya citadas, el tomismo sí ve complementariamente el desarrollo teórico y la narración al incluir en su narrativa a sus rivales, relatando la historia de sus contrincantes dentro de su propia historia (MacIntyre, 1990: 113). De esta manera, MacIntyre sigue el lineamiento realista y finaliza su proyecto narrativo de restauración epistemológica de la ética que había comenzado en After Virtue, al concluir que la Tradición de las virtudes es racionalmente superior a las otras dos.

\footnotetext{
${ }^{4}$ Luego, en Intractable Moral Disagreements (2009) va a identificar el utilitarismo como una tradición más y narrará allí su historia, aunque al concluir su proyecto renovación epistemológica en 1990, termine desarrollando sólo las primeras tres tradiciones.
} 


\title{
Segunda cuestión:
}

¿Cómo exhibe un agente la racionalidad y justifica sus juicios a través de una narrativa? a) ¿Por qué en Ethics in the Conflicts of Modernity el autor se centra más en el concepto de narrativa y no tanto en el de Tradición?

\author{
En Whose Justice? Which Rationality? (1988) MacIntyre afirmaba que cada artículo de la Suma \\ Teológica de Santo Tomás de Aquino

\begin{abstract}
"plantea una pregunta cuya respuesta depende del resultado de un debate esencialmente incompleto. Porque el conjunto de argumentos heterogéneos y a menudo disparatados en contra de cualquier postura a la que las investigaciones del Aquinate por el momento le habían conducido a aceptar siempre está abierto a añadiduras de argumentos aún no previstos. Y no hay modo alguno, por tanto, de excluir por adelantado la posibilidad de que aquello hasta ahora admitido tenga que modificarse o incluso rechazarse. Aquí no hay nada peculiar en los procedimientos del Aquinate. Es de la naturaleza de toda dialéctica, entendida tal como Aristóteles la entendía, ser esencialmente incompleta” (p. 175).
\end{abstract}

De acuerdo con este modo de proceder realista, en el año 2016, veintiséis años después de concluir su proyecto unitario de restauración epistemológica, MacIntyre decidió reanudar su narración en lo tocante a la teoría moral con la publicación de Ethics in the Conflicts of Modernity. An Essay on Desire, Practical Reasoning and Narrative. Esta vez nuestro autor, con el mismo afán de superar los desacuerdos morales, se propone hacerlo a partir de un diálogo únicamente entre dos tradiciones: el expresivismo, cuyo antecedente es el emotivismo, y la tradición tomista aristotélica. La óptica adoptada en esta reciente obra es la de la psicología, retomando temas presentes en Dependent Rational Animals (1999), además de incluir temas relacionados con la sociología, la economía y la política, a la luz del estudio de la incidencia del deseo en el razonamiento práctico.

MacIntyre señala que las disciplinas académicas están estructuradas de forma que la filosofía, la sociología y la historia, determinantes de la vida práctica y moral, no se integren. De ahí la necesidad imperiosa de recurrir a la narrativa para comprenderlas acabadamente. Uno de los propósitos de su investigación es invitar al lector a vivir 'contraculturalmente', contra la ética del mercado y la política estatal, producto de la Moral moderna, a partir de las ideas del tomismo y el aristotelismo con algunos aportes de Marx.

Con tan sólo prestar atención al índice del libro, ya se puede advertir que MacIntyre adopta la dinámica de la dialéctica tomista en los primeros cuatro capítulos planteados como quaestiones. En consecuencia, en el primer capítulo plantea el problema, por ejemplo, ¿qué son los deseos y qué significa tener buenas razones para desear algo? En segundo lugar, expone argumentos en contra, en este caso, los del expresivismo. En tercer lugar, presenta argumentos a favor de su postura, los de Aristóteles. En cuarto lugar, reformula la postura tomista aristotélica para fundamentar su propia postura narrativista. Por último, presenta soluciones a problemas planteados en contra por parte del expresivismo. Así es como MacIntyre entiende que funciona una tradición viva, y este movimiento se da en el tiempo, con determinado orden y con una alta carga moral por tratarse de una actividad dirigida a la consecución de la verdad con vistas al bien. Esto posibilita que se constituya una trama narrativa que represente cómo esta tradición va desarrollándose en el curso temporal.

Ahora bien, al comienzo de este trabajo nos propusimos explicar la razón por la cual MacIntyre, después de tantos años, sigue profundizando en el tema de la narrativa y no presta tanta atención a un tema que había sido central en su pensamiento: la Tradición. Una de las razones posibles es la noción de lo que él llama 'narrativa justificativa teórica'. La narrativa justificativa teórica se utiliza para argumentar frente a los filósofos de la moral, mientras que para responder a las preguntas del agente, se recurre a una justificación práctica.

En el ámbito de lo teórico, para justificar una posición frente a la maldad o bondad de acciones determinadas se recurrirá, por ejemplo, a la exposición del esquema tomista de la teoría de la acción, pasando de las tesis coyunturales a las más fundamentales. "Pero si este esquema es cuestionado, la justificación deberá tomar una forma distinta. Los argumentos, en tal caso, deberán presentarse en 
forma de una narrativa histórica, que comience con las respuestas de Aristóteles a sus predecesores, pasando de ahí a pensadores presocráticos, a Sócrates y a Platón” (MacIntyre, 2017: 348). Luego, el replanteamiento radical del aristotelismo de parte de Santo Tomás de Aquino, para continuar hacia los problemas planteados sucesivamente por la nueva comprensión de la ley y la moral que encuentra expresión en la creación del Estado moderno, por la nueva ciencia natural anti-aristotélica, por la Ilustración, y por la filosofía post-kantiana. "Puesto que la dimensión teórica requiere una historia compuesta por sucesivas etapas críticas en el desarrollo de posiciones y argumentos teóricos, adopta la forma de una narración en tercera persona que discurre hasta alcanzar el presente" (MacIntyre, 2017: $350)$.

De esta forma, los tomistas han de ser capaces de reconocer aquellas objeciones que constituyan una razón suficiente para rechazar las tesis fundamentales del realismo. Y una vez que hayan sido falsadas, se podrá afirmar, siguiendo a C. S. Pierce, que son ciertas. Por lo tanto si, tras considerarlo debidamente, los tomistas descubren que tienen buenas razones para rechazar las objeciones aducidas por sus críticos, lo que resta es investigar los errores de los críticos y por qué estos argumentos resultan convincentes.

A partir de estas disquisiciones, podríamos llegar a sostener que la tarea de la narrativa justificativa teórica MacIntyre la ha realizado en el corpus conformado por After Virtue, Whose Justice? Which Rationality? y Three Rival Versions of Moral Enquiry, en cuyo desarrollo fue necesario explicar y desarrollar con detenimiento la noción de Tradición de investigación racional. Y si bien hay cierta continuidad de esta narrativa teórica en Ethics in the Conflicts of Modernity, especialmente en los capítulos II y III, su propósito es persuadir no sólo al teórico, sino también al agente que se pregunta cómo actuar correctamente, aunque la cultura dominante intente redirigir sus deseos hacia otra parte.

\section{b) ¿Por qué MacIntyre considera que la narratividad es inherente a la justificación racional de los agentes tomistas aristotélicos?}

En el primer capítulo de la obra que nos ocupa, MacIntyre se pregunta cuáles son las condiciones de posibilidad humanas para alcanzar lo bueno y lo mejor. Cabe señalar que aquí hace una oportuna referencia al lenguaje propio de la naturaleza racional del hombre. El lenguaje hace posible no sólo la comunicación, sino también contemplar futuros alternativos, narrar historias, aprenderlas y representarlas. "And fourthly, these same linguistic resources enable us to tell each other stories about our projects, our heroic enterprises and our tragic failures, stories that we narrate, stories that we enact, stories that we sing, stories from which we learn" (MacIntyre, 2016: 27) ${ }^{5}$.

En el segundo capítulo, el escocés afirma que los agentes prácticos reflexivos se vuelven autoconscientes de tres formas: En primer lugar, explicitan y dilucidan conceptos y tesis cuyo rango de aplicación y verdad han presupuesto sin ser conscientes de ellos. En segundo lugar, descubren que las realidades de la vida práctica quedan recogidas en narraciones sobre el fracaso y el éxito como razonadores prácticos al ejemplificar la fronesis o la prudentia. Finalmente, se hacen autoconscientes del fin al que tienden (aunque no puedan decir mucho de él) por cómo se van inclinando hacia él en sus vidas (MacIntyre, 2016: 74).

Ya indicamos en el apartado anterior que en el debate con los teóricos rivales, los tomistas aristotélicos recurren a la narrativa justificativa teórica. Pero en caso de que la discusión se dé con un agente que nos hace preguntas prácticas, la justificación "adopta la forma de una historia particular contada por cada agente particular sobre sí mismo, es desde el inicio una narración en primera persona, aunque esa primera persona sea a veces un 'yo' y a menudo un 'nosotros"' (MacIntyre, 2017: 350).

\footnotetext{
${ }^{5}$ Es lamentable que en la versión castellana de esta obra el traductor haya omitido expresiones fundamentales del autor en las que reafirma su interés por la narrativa.
} 
Esto es lo que MacIntyre denomina narrativa justificativa práctica y constituye una novedad en el narrativismo macinteryano. Ella consiste en narrar la historia de las potencialidades racionales del agente y de cómo aprendió a priorizar bienes. Un punto de vista típico de esta forma de justificación es la experiencia de una quiebra en la vida de alguien, cuando surge un problema y aparecen las incompatibilidades. Un ejemplo de esta situación lo podemos encontrar en la propia biografía intelectual de MacIntyre, que pasa de ser un miembro del partido comunista a convertirse al catolicismo. Su comprensión de la felicidad y su concepto de bien cambian completamente. "Lo que aporta este segundo tipo de narración es la mejor justificación que por el momento puede ofrecer el agente particular que narra, según los mejores estándares que el agente tiene ahora a su disposición para realizar sus juicios prácticos" (MacIntyre, 2017: 352).

El escocés aclara que muy frecuentemente los agentes que justifican sus acciones de esta forma, propia del tomismo aristotélico, no conocen los desarrollos ni de Aristóteles ni de Santo Tomás. Es por eso que no se van a remitir a una teoría sino a la historia de su propia vida para dar cuenta de sus razones para actuar, para reconocer sus propios errores y para identificar los bienes que están en juego.

Finalmente, en el cuarto capítulo, MacIntyre alude a la importancia del ejercicio de las virtudes en términos tomistas aristotélicos. La calidad humana o institucional, afirma, se mide por la práctica de las virtudes en orden al bien común, esto es, la causa final de la vida política. Así, al momento de evaluar individuos o instituciones, se hará en función de las virtudes por parte de los individuos en sus roles sociales, como miembros de familias, profesores, bedeles, empleados, etc., y la orientación general de la institución. "Evaluar de este modo es presuponer una comprensión narrativa tanto de los agentes individuales como de las instituciones, presuponer que es solo en los contextos que aportan las narraciones de fondo que las acciones concretas y los cursos de acción pueden ser adecuadamente entendidos y evaluados" (MacIntyre, 2017: 368).

\section{c) ¿Cuál es el argumento último que legitima la narratividad como el principio de inteligibilidad de las acciones humanas?}

MacIntyre afirma que el fin último, el telos, es el fundamento de la unidad narrativa de la vida humana porque constituye nuestras vidas como un todo.

\footnotetext{
"Cuando actuamos para conseguir algún bien concreto, también actuamos para conseguir nuestro fin último, y es esto lo que hace que nuestras vidas tengan, si actuamos correctamente, una orientación hacia ese fin. Es por esta razón-y aquí, por supuesto, estoy yendo más allá de Tomás de Aquino (algunos tomistas dirían que más bien me alejo de él)--, que nuestras vidas tienen la estructura narrativa que tienen, y que sólo podemos hacernos adecuadamente inteligibles en términos narrativos" (MacIntyre, 2017: 386).
}

Según nuestro juicio, nuestro autor no se contradice con el Aquinate.

Podríamos afirmar, entonces, que porque hay un fin último, hay un comienzo y un desarrollo que configuran la trama narrativa. MacIntyre sostiene que a medida que en nuestras vidas nos movemos hacia el fin último, cada uno de nosotros representa una historia que tiene, según dice Aristóteles, una serie de finales posibles, dependiendo de la combinación entre eudaimon, makarios (disfruta de los logros y de la buena fortuna) o athlios (infeliz, desdichado, contrariado). "Lo que Aristóteles excluye respecto de los fines--, no así Tomás de Aquino, es la posibilidad de que se den situaciones en las que no conseguir alcanzar ciertos objetivos finitos, sin importar cuán grandes sean, no constituya una señal de fracaso" (MacIntyre, 2017: 386).

Finalmente, cabe señalar la diferencia entre la comprensión narrativa de los expresivistas y la de los aristotélicos. Si comparamos las narraciones de los expresivistas y las de los neoaristotélicos, en las historias de los primeros abundan las referencias a los afectos, incluso la racionalidad se considera expresión de ciertos sentimientos y lo más relevante es lo que le importa a la persona. En cambio, para los neoaristotélicos, lo más importante son los razonamientos prácticos en orden a la consecución del fin último, y el fracaso o el éxito en este propósito. Entre ambas corrientes hay tres niveles de disenso: 
En primer lugar, hay discrepancias acerca de cómo resolver el conflicto entre juicio y deseo. En segundo lugar, se diferencian en cómo interpretar los juicios acerca de lo que es bueno hacer en determinada situación. Por último, difieren en la manera en que la historia de un agente práctico debe ser relatada para hacerse entender.

Antes de cerrar este trabajo, en función de la extensión permitida cabe, sin embargo, señalar que no trataremos las posturas contrarias al narrativismo desarrolladas por el autor en esta obra respecto de las posiciones de Sartre, Máirtin Ó Cadhain, Kafka, Tarantino, Strawson y Tengelyi. Por las mismas razones, no abordaremos el análisis de las cuatro narraciones de vidas de razonadores prácticos ejemplares presentadas por el autor.

\section{Conclusiones}

Después haber hecho un breve recorrido histórico sobre las primeras formulaciones de MacIntyre acerca del narrativismo, en las que sostiene que las acciones humanas se hacen inteligibles por medio de las narraciones y que la investigación racional es inteligible en términos de tradiciones, nuestra atención se ha focalizado en la reaparición de la problemática de la narratividad.

En esta nueva versión, aparecida veintiséis años después, hemos observado por un lado, una continuidad con las postulaciones anteriores. Por otro lado, advertimos una preponderancia de la problemática de la narratividad sobre la de la tradición, para centrarse en la justificación racional que se desarrolla narrativamente, y se ordena al telos el cual se constituye como el fundamento que legitima la narratividad como el principio de inteligibilidad de las acciones humanas.

Respecto de su modalidad expositiva, cabe destacar la formalización de los capítulos a la manera de las quaestiones tomistas, de ahí la presencia de la compulsa con numerosos autores entre los que se cuentan no sólo filósofos sino también economistas, politólogos, sociólogos, psicólogos, literatos y cineastas. Como cierre, y fuera del tratamiento que hemos dado a esta obra, merece la pena destacar que el autor se convierte él mismo en narrador -biógrafo_ de la vida de cuatro personajes reales.

En suma, lo que hace MacIntyre en Ethics in the Conflicts of Modernity, la más reciente de sus obras, es postular una teoría moral siguiendo el orden de la inteligencia humana. Lo hace no sólo movido por la búsqueda de la verdad en el orden teorético, sino también con el afán caritativo de descubrir y hacer descubrir, con empatía, paciencia, generosidad y humildad en qué consiste la felicidad verdaderamente y por qué vías - narrativas - alcanzarla.

\section{Referencias}

Hortal, A. (1998). "MacIntyre y la crítica comunitarista a la modernidad". Relaciones. Revista al tema del hombre. Serie Filósofos de hoy II. no. 173. Recuperado de http:/www.chasque.net/frontpage/relacion/9810/filosofos_de hoy.htm

Knight, K. (1998). The MacIntytre Reader. Cambridge: Polity Press.

Lorenzo, I. (2007). Comunitarismo contra individualismo. Una revisión de los valores de occidente desde el pensamiento de Alasdair MacIntyre. The Global Law Collection. Pamplona.

MacIntyre, A. (2006). "Epistemological Crises, Dramatic Narrative, and the Philosophy of Science. The Monist". (1977). 60. no. 4. reimpreso en: MacIntyre, A. The Tasks of Philosophy. Cambridge University Press. Cambridge.

MacIntyre, A. (1981). After Virtue. A Study in Moral Theory. University of Notre Dame Press, 2nd edn. 1984, 3rd edn. 2007. Notre Dame. Traducido como Tras la virtud (2004). Crítica. Segunda edición en castellano. Barcelona. 
MacIntyre, A. (1988). Whose Justice? Which Rationality? University of Notre Dame Press. Notre Dame. Traducido como Justicia y racionalidad. (2001). Eiunsa. segunda edición en castellano. Madrid.

MacIntyre, A. (1990). Three Rival Versions of Moral Enquiry. Enciclopaedia, Genealogy and Tradition. University of Notre Dame Press. Notre Dame. Traducido como Tres versiones rivales de la ética. Enciclopedia, Genealogía y Tradición. (1992). Rialp. Madrid.

MacIntyre, A. (1999). Dependent Rational Animals: Why Human Beings Need the Virtues. Open Court. Chicago.

MacIntyre, A. (2016). Ethics in the Conflicts of Modernity. An Essay on Desire, Practical Reasoning and Narrative. Cambridge University Press, Nueva York. Versión en castellano: (2017) Ética en los conflictos de la modernidad. Sobre el deseo, el razonamiento práctico y la narrativa. RIALP. Madrid. 